\title{
A protocol for the development of a Core Outcome Set for PREgnancy Nutrition (PRENCOS)
}

\author{
Sarah Louise Killeen, Dr. Eilleen C. O’Brien, Dr. Aisling A. Geraghty and \\ Prof. Fionnuala M. McAuliffe \\ UCD Perinatal Research Centre, School of Medicine, National Maternity Hospital, University College Dublin, \\ Dublin 2, Ireland
}

\section{Abstract}

Introduction: Maternal nutrition during pregnancy is a modifiable risk factor for health. Heterogeneity in outcome reporting in studies evaluating nutrition in pregnancy limits their comparability and is a barrier for high quality evidence synthesis. A core outcome set (COS) is set of outcomes, which are agreed by consensus, to be a minimum standard to report within an area of research. The CoRe Outcomes in Women's and Newborn health (CROWN) initiative is an international initiative which supports the development of COSs for Women and Newborn health research. To date, there is no COS which specifically addresses the issues with outcome reporting in research on nutrition in pregnancy. Therefore, we present a study protocol for the development of a COS for maternal nutrition research in pregnancy.

Methods: The COS is registered with the Core Outcomes for Measurement of Effectiveness Trials (COMET) registry. A systematic review will be conducted following PRISMA guidelines to identify studies evaluating maternal nutrition during pregnancy. Outcomes will be extracted from eligible studies and cataloged using the taxonomy of the COMET initiative. We will make efforts to supplement our findings with outcomes from other sources, including qualitative interviews with mothers. Secondly, a modified Delphi survey will be conducted with international stakeholders including healthcare professionals, researchers and mothers. Participants will be presented with the list of outcomes from step one and invited to rank the importance of including each outcome in the final COS using a 9-point Likert scale. We will complete descriptive statistics and in a second round of the survey, participants will receive feedback on their individual scores from round one, along with the average score each stakeholder group provided for each outcome. Based on this, participants will have the opportunity to change their scoring and will be encouraged to give a rationale for their final choice. Lastly, a face-face consensus meeting will be held with representatives from all stakeholder groups to finalise the COS.

Results: We will identify the outcomes reported in maternal nutrition research. We will also determine the outcomes which are important to pregnant women and if these are missing from the literature. A final COS for nutrition research in pregnancy will be developed.

Discussion: This COS will support the harmonisation of outcome selection and reporting in maternal nutrition research which is necessary for high quality evidence synthesis to support clinical practice and the nutritional care of pregnant women.

\section{Conflict of Interest}

There is no conflict of interest 